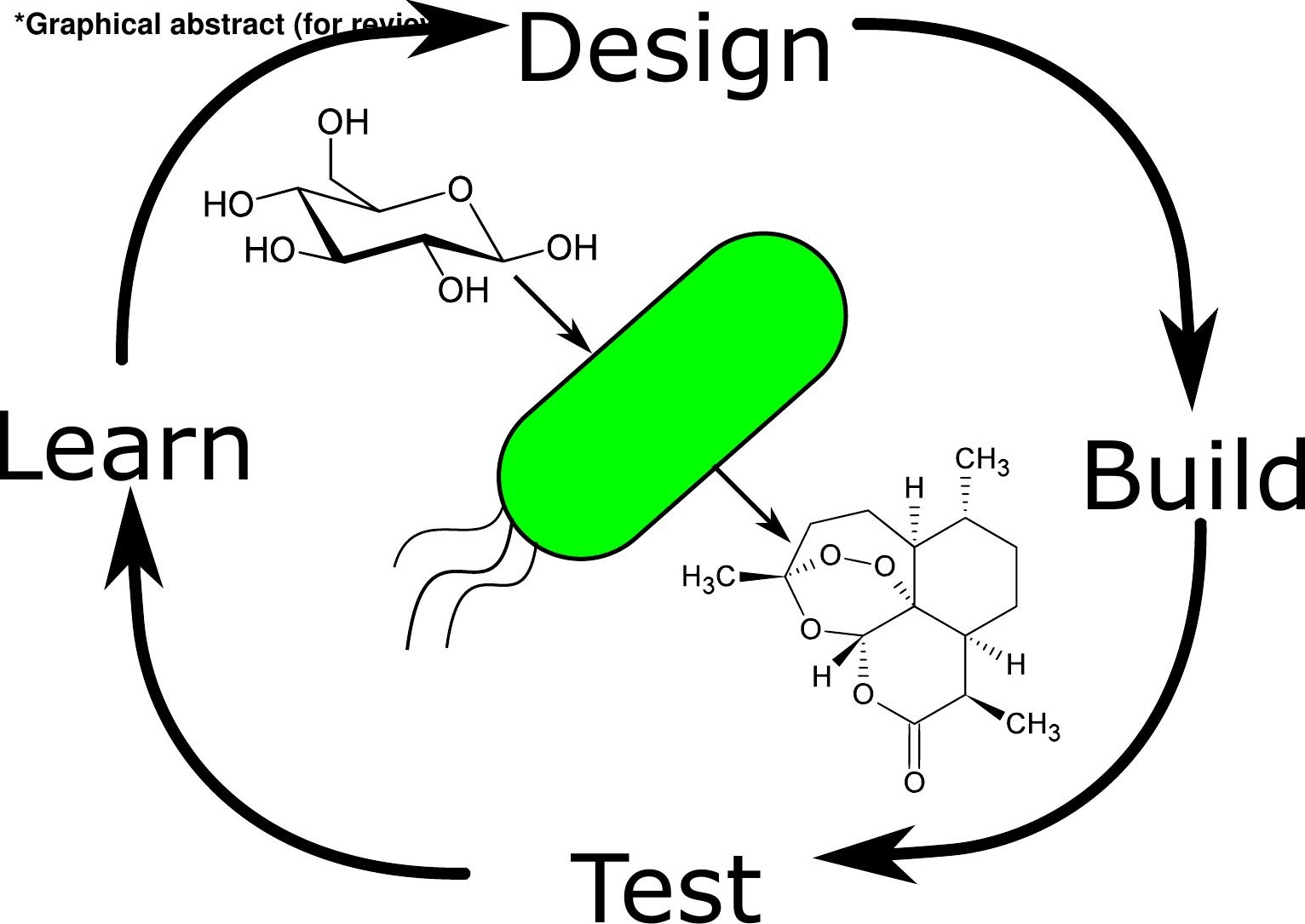




\title{
Complex Systems in Metabolic Engineering
}

\author{
James D. Winkler ${ }^{\mathrm{a}}$, Keesha Erickson ${ }^{\mathrm{a}, 2}$, Alaksh Choudhury ${ }^{\mathrm{a}, 2}$, Andrea L. \\ Halweg-Edwards ${ }^{\mathrm{a}}$, Ryan T. Gill ${ }^{\mathrm{a}, 1}$ \\ ${ }^{a}$ Department of Chemical and Biological Engineering, University of Colorado-Boulder, Jennie \\ Smoly Caruthers Biotechnology Building, Research Park, Boulder, CO USA 80303
}

\section{Abstract}

M 1 and often unknown interconnectivity between and within these networks, often prevents researchers from achieving desired performance. Other fields have developed methods to tackle the issue of complexity for their unique subset of engineering problems, but to date, there has not been extensive and comprehensive examination of how metabolic engineers use existing tools to ameliorate this effect on their own research projects. In this review, we examine how complexity affects engineering at the protein, pathway, and genome levels within an organism, and the tools for handling these issues to achieve high-performing strain designs. Quantitative complexity metrics and their applications to metabolic engineering versus traditional engineering fields are also discussed. We conclude by predicting how metabolic engineering practices may advance in light of an explicit consideration of design complexity.

Email addresses: james.winkler@colorado.edu (James D. Winkler), keesha.erickson@colorado.edu (Keesha Erickson), alaksh.choudhury@colorado.edu (Alaksh Choudhury), andrea.edwards@colorado.edu (Andrea L. Halweg-Edwards)

${ }^{1}$ Corresponding author. Tel: (303) 492-2627, email: rtg@colorado.edu.

${ }^{2}$ These authors contributed equally to the manuscript. 


\section{Introduction}

$14 \quad$ Metabolic engineering has undergone profound changes from its initial origins in bioprospecting [1], random mutagenesis, and screening. Today, our increasingly large body of knowledge and powerful tools have enabled the creation of a vast array of microbial cell factories [2]. Capabilities continue to accelerate due to advances in underlying technologies: automated genome engineering [3], high-throughput genome modification [4], and recombineering [5]. As such, future approaches like whole genome synthesis [6]will provide new levels of control over strain performance. However, similarly rapid improvements in our ability to comprehend what constitutes a successful design that responds to environmental cues effectively and produces the desired performance are lacking [7]. The majority of metabolic engineers still rely on human inference when generating new strains (Figure 1), which typically entails manual examination of metabolic pathways and designs drawn from the personal experience of the experimenter, rather than a defined design algorithm. This approach is not feasible for future large-scale strain construction efforts based on continued advances in DNA synthesis, which will enable the creation of tens of thousands of made-to-order strains modified at the genome-scale. Rather, new forward engineering approaches that automate strain design, construction, and testing while invoking

machine learning and related computational techniques are required.

The core challenge to the development of such approaches is the fundamental lack of understanding of the rules governing the design of complex biological systems. While there have been significant developments in computational strain design methodology, we still are unable to accurately predict design performance at the de- 
gree of accuracy required, as has been noted recently [8]. Creating and manipulating complex systems, however, is a fundamental aspect of almost all engineering fields. For example, as software systems grew beyond a size comprehensible by a single person, researchers began to create formal methodologies and analysis tools to aid in complexity abstraction, enabling productivity gains to continue apace. Most nontrivial software packages today are developed by large teams, where no one individual has the responsibility for (or perhaps knowledge of) the entire codebase. Analogous to the plethora of integrated software development environments, analysis packages, and libraries available to software engineers, similar support tools are under active development by metabolic engineers to improve our ability to cope with the inherent complexity of living organisms and the many unknown variables influencing their behavior.

Given the importance of complexity in the context of metabolic engineering, this review aims to examine several aspects of systems complexity that metabolic engineers commonly grapple with during the strain development process, along with possible ways of reducing its impact on project development. We focus on how complexity impacts three distinct areas that engineers commonly manipulate to improve chemical production: protein engineering, improving or altering enzymes to manipulate an individual facet of metabolism; pathway construction, channeling flux into the desired branches of the metabolic network; and genome engineering, ensuring that all of these modifications interoperate efficiently. Finally, the quantification of complexity and its implications for the practice and scale of metabolic engineering are examined. 


\section{Protein-level Engineering}

Much like individual methods are the building blocks for software, the first level metabolic engineers can target for strain improvement are the enzymes responsible for substrate conversion. Design parameters at this level include protein expression, activity, stability, target specificity, and a range of others characteristics [9]. Several methods with different knowledge requirements (i.e. complexity) have been developed to assist in this process (Figure 2). Various types of transcription and translation optimization exist, depending on the needs of the experimenter, such as controlling plasmid copy number [10], codon usage [11], promoter strength [12], mRNA stability, and RBS efficacy [13]. These parameters can be changed using a reverse-engineering approach that requires little knowledge of the desired expression goal, or cellular models can be applied to estimate the optimal expression level for the protein of interest. These methods represent a trade-off between experimental effort via knowledge-based approaches, with the former representing a low complexity (in terms of data required) tactic but necessitating reverse engineering versus high complexity model driven analyses that require substantial empirical testing, computational development and validation, but can provide physical or statistical bases for engineering suggestions. This classification can be extended beyond expression optimization to sequence-level alterations in the protein itself.

The contrast between high complexity data-driven and low-complexity random approaches extends to improving protein function by mutation. Reverse engineering approaches, like error-prone PCR and DNA shuffling, have proven to be highly effective in improving properties such as activity, substrate range, and stability when 
structural or functional information is unavailable [14]. These techniques have the added advantage of low up-front cost and knowledge required for generating and screening target libraries. As with other library-based approaches, mutagenesis methods can be limited by complex fitness landscapes [15] that arise from epistatic interactions between residues, the screening effort required for large libraries, and subsequent investigation into mechanism improving function. These limitations are inherent to all random approaches, as it is often difficult to understand how phenotypes are improved through mutagenesis without a conceptual structure-function model. Random methods are, as a result, of generally low conceptual complexity, but their explanatory power can be limited without extensive reconstruction and physical modeling.

In contrast to random mutagenesis, forward approaches to protein engineering are able to reduce the experimental effort by identifying residues of interest at the cost of substantially increased algorithmic complexity. A combination of structural analysis, bioinformatics and de novo protein design tools have been quite successful in developing improved biocatalysts [16]. Structure-activity methods such as proSAR [17] require previously generated data to identify key residues that affect protein function, although the model in this case is purely statistical and lacks the complexity inherent in physical protein models. For proteins with known structures, tools such as Rosetta [18] and SCHEMA [19] reduce library size (trading algorithmic complexity to reduce experimental burden) by eliminating unstable mutants for site saturation mutagenesis and site directed chimeragenesis, respectively. Such a combination has proven effective in metabolic engineering designs [20-23]. The key 
unifying factor behind all these tools is a reduction in experimental effort relative to random approaches. Despite the high initial cost of developing high complexity methods, these tools can significantly reduce the time, effort, and labor associated with classical protein engineering tools.

\section{Pathway-level Engineering}

The same reverse versus forward engineering complexity trade-off also applies at the pathway level. Complexity increases when engineering a pathway, as the number of engineering parameters expands to include interactions among various pathway enzymes and overall cellular metabolism (Figure 3). The key design parameter metabolic engineers must contend with is gene selection and a number of in silico tools have been developed to assist researchers in selecting the best gene(s) for their pathway based on thermodynamics, pathway length, and other criteria (see [24] for an extensive review).The Biochemical Network Integrated Computational Explorer (BNICE) is one of the most well-known examples of these tools [25]. Additional enzyme selection tools include Metabolic Tinker [26], FMM [27], and Desharky [28]. For retrosynthetic approaches, the user selects an end product and pathways to that product are suggested [29-31]. Future tools may be able to predict whether enzymes can operate on non-natural substrates along with accounting for metabolite toxicity issues as well, reducing the difficulty of pathway design further. No matter the underlying implementation or selection criteria, these tools all allow researchers to abstract the complexity of enzyme selection by employing various modeling techniques.

Once the pathway has been specified, it must be optimized for maximum per- 
formance. The increased number of design parameters at this stage, including the relative expression levels, activities, environmental tolerance, and other properties, represent a significant increase in complexity compared to the engineering of a single protein. Both reverse and forward engineering techniques can be applied to grapple with this problem; examples for the former include high-throughput library generation and screening (or selection) [32-34], global regulatory engineering [35, 36] or combinatorial module optimization [37]. Reverse engineering is necessary in particular when gaps in knowledge present additional complexity; for instance, with limited knowledge, gene expression studies can deliver insight into pathway regulation [38, 39], while metabolomics and meta-metabolomics profiling can lead to the discovery of novel biological products [40]. Reverse engineering approaches rely on generation and screening of many potential production strains in order to identify optimal parameter sets post hoc, thereby exchanging screening effort for up-front system modeling. Forward engineering is exemplified by the many computational pathway and metabolic models that guide metabolic interventions [41-43], or those that exploit thermodynamic modeling to create irreversibilities in pathway flux [44]. The distinguishing factor between forward and reverse pathway engineering is that forward methods attempt to model sufficient parameters to predict favorable pathway interventions, and require a substantial knowledge base concerning both the organism and enzymes in question. 


\section{Genome-level Engineering}

Engineering an entire organism poses challenges beyond those commonly encountered at the protein or pathway levels, as described in Figure 4. The number of design parameters increases significantly, as was the case from the protein to pathway transition: redox balance must be maintained [45], flux must be maximized through common metabolic checkpoints [46], and products must be sufficiently exported out of the cell to minimize toxicity [47], among many other parameters. Forward engineering of desirable phenotypes becomes more challenging as a result, and reverse engineering is often used to improve industrially relevant phenotypes due a lack of knowledge concerning the underlying molecular mechanisms for a set of desirable traits. In the absence of a known genotype-phenotype connection, non-targeted methods such as adaptive evolution, genome-scale libraries, and other tools can effectively improve industrially relevant phenotypes $[4,48,49]$, including production of specific metabolites [50]. Other reverse engineering methods can be applied to individual proteins, such as product-export pumps, to avoid toxicity and increase thermodynamic driving forces on production [51]. Designing self-regulating strains can also ameliorate complexity, as there is no need to manually regulate pathway expression without external inducers to both minimize toxicity and maximize productivity [36]. These approaches reduce the knowledge required for achieving the desired production phenotype, thereby reducing the complexity of genome-scale engineering compared to forward approaches, such as metabolic modeling.

True forward design of an entire genome, in contrast to reverse engineered alterations, is currently beyond the capabilities of metabolic engineers. Gibson et al. 
took the first steps towards this goal by constructing the first chemically synthesized bacterial genome [6]. Dymond et al. have undertaken a related effort to replace yeast chromosome with redesigned analogs as well [52]. However, rational approaches to engineering organisms at this scale will require dramatic reductions in DNA synthesis and sequencing costs, as well as an improved understanding of the minimal sequence requirements for engineering a chassis genome. These efforts will simultaneously rely on and improve whole cell models due to their generation of large genotypephenotype datasets. The powerful whole-cell model of Mycobacterium by Karr et al. [53] and the original E-CELL model [54] demonstrate some of the physiological insights, including estimates of protein-DNA binding frequency, that can be gleaned from this approach. However, due to the much larger genome size of most common metabolic engineering platform strains, developing realistic and computable whole cell models for these organisms poses additional challenges. Both genome redesign and cell modeling efforts represent the forefront in metabolic engineering complexity, as interactions at all three levels discussed here must be explored in order to build made-to-order biocatalysts.

Although the field of genome design is in its infancy at the moment, we expect that future developments in DNA synthesis, cloning, and automated re-engineering of genome architectures will lead to the gradual introduction of dramatically rewired organisms that should exhibit reduced complexity-related issues. However, since even simplistic organisms require sophisticated regulatory and metabolic networks for growth and efficient catalysis, grappling with complexity through a combination of experimental validation, simulation, and modularization will remain a challenge 
at the forefront of metabolic engineering at all of the scales explored here.

\section{Measuring Complexity}

It will soon be possible to generate a comprehensive library of genome-scale designs in a single experiment, rather the relatively small-scale projects that currently characterize most metabolic engineering efforts. Taking advantage of this explosion in the number of testable designs is extremely challenging, as the subsequent experimental design evaluation and analysis steps remain comparatively slow [55]. Complexity assessment can be used to address important aspects of this issue, as filtering high-complexity designs from this pool of constructs could significantly reduce the downstream screening burden. We therefore examine two representative examples of complexity metrics that could potentially be adapted by metabolic engineers for this purpose: one that is primarily derived from network topological analysis and another assessing the breadth and depth of the language used to describe a software program. An extensive review of a range of other metrics in the context of software engineering is given by Zuse [56].

Emblematic of software topological analysis is McCabes cyclomatic complexity (CC, [57]) which assesses the complexity of control flow in a software program by representing functions as nodes and control logic connecting them as edges. A large $\mathrm{CC}$ value implies more extensive control logic than other programs, while a small $\mathrm{CC}$ value indicates that few branch points occur during data processing. However, this approach assumes that both the underlying network topology is human-defined and the flux between nodes is one of information, rather than mass, as is the case for 
metabolic networks. Given the small number of modified genes in most metabolic engineering designs (Winkler et al., in press), the changes in CC value for most designs compared to the base network will be minute. It may be possible to examine only small, heavily manipulated network modules to calculate a more meaningful topological complexity score.

A non-topological alternative to assessing complexity can be found in the Halstead metric [58]. This approach examines the number of unique operators (methods for acting on variables) and operands (variables) used in a program, and how many times these are used to define the volume (size), difficulty, and expected amount of programmer effort required for implementation. In the context of metabolic engineering, operators are roughly analogous to genetic tools for introducing mutations into strains, while operands can be taken as manipulated genes. Expanding the definition of Halsteads metric to include other project facets, such as the motivation behind introducing specific mutations and the number of metabolic network elements manipulated as a consequence of genetic modifications, may be appropriate to better describe the complexity to a given design.

Both volume and topology-based metrics have had success in improving several aspects of software engineering. The most important application has been estimating and reducing software maintenance costs [59,60], as these comprise $40-70 \%$ of the total lifecycle cost of a program [59]. Assessment of the ability of software engineers to identify programming errors has also a notable application of these complexity metrics [61,62]. Security issues can also be anticipated by identifying highly complex (i.e. error prone) modules within a software engineering project, allowing costly 
vulnerabilities to be identified and corrected before they are exploited post-release [63]. Although these applications are unique to software engineering, it is simple to envision analogous roles complexity metrics may play in the context of metabolic engineering: discriminating against high-complexity designs that are difficult to optimize by accounting for protein, pathway, and genome alterations, evaluating the effect of a library design on the host transcriptome and metabolic network, and estimating the overall cost of metabolic engineering research projects. As the necessary standards, data sources, and computational infrastructure become available, we expect that metabolic engineers will begin to explore these applications and more.

\section{Conclusions}

In this review, we have examined common sources of complexity at each cellular level and two potential routes for quantifying complexity. Given the increasing interest in developing organism foundries and standardizing organism engineering, efficient filtering of potential designs to identify the least difficult to implement with the highest chance of meeting the desired productivity level is becoming increasingly necessary. Winnowing the set of all possible designs via complexity filtering complements the rapid advances in genome engineering technologies by allowing researchers to eliminate designs that are challenging to construct and optimize without experimental screening. As metabolic engineering becomes increasingly standardized, complexity analysis tools and guidelines will become an integral part of our engineering toolbox. 


\section{Acknowledgments}

This project was financially supported by the Department of Energy Genome Sciences Program (award \#: DE-SCOOO8812) and (other funding sources). We also thank Gur Pines for helpful suggestions on the manuscript. 


\section{References}

\section{References}

[1] Pthe activation of the butanol-acetone fermentation of carbohydrates by Clostridium acetobutylicum.

[2] David Jullesson, Florian David, Brian Pfleger, and Jens Nielsen. Impact of synthetic biology and metabolic engineering on industrial production of fine chemicals. Biotechnology Advances, 2015.

[3] Peter A Carr and George M Church. Genome engineering. Nature Biotechnology, 27(12):1151-1162, 2009.

[4] Joseph R Warner, Philippa J Reeder, Anis Karimpour-Fard, Lauren BA Woodruff, and Ryan T Gill. Rapid profiling of a microbial genome using mixtures of barcoded oligonucleotides. Nature Biotechnology, 28(8):856-862, 2010.

*This study develops a genome-wide approach for rapidly modifying expression levels of all genes simultaneously, allowing the study of how each gene impacts fitness under a wide variety of conditions of interest.

[5] Gur Pines, Emily F. Freed, James D. Winkler, and Ryan T. Gill. Bacterial recombineering - genome engineering via phage-based homologous recombination. ACS Synthetic Biology, 2015. PMID: 25856528.

[6] Daniel G. Gibson, John I. Glass, Carole Lartigue, Vladimir N. Noskov, RayYuan Chuang, Mikkel A. Algire, Gwynedd A. Benders, Michael G. Montague, 
Li Ma, Monzia M. Moodie, Chuck Merryman, Sanjay Vashee, Radha Krishnakumar, Nacyra Assad-Garcia, Cynthia Andrews-Pfannkoch, Evgeniya A. Denisova, Lei Young, Zhi-Qing Qi, Thomas H. Segall-Shapiro, Christopher H. Calvey, Prashanth P. Parmar, Clyde A. Hutchison, Hamilton O. Smith, and J. Craig Venter. Creation of a Bacterial Cell Controlled by a Chemically Synthesized Genome. Science, 329(5987):52-56, 2010.

** The authors construct and transform the first completely synthetic bacterial genome and demonstrate its functionality in Mycobacterium.

[7] Evangelos Simeonidis and Nathan D Price. Genome-scale modeling for metabolic engineering. Journal of Industrial Microbiology and Biotechnology, pages 1-12, 2015.

[8] Michael Bölker. Complexity in Synthetic Biology: Unnecessary or Essential? In Synthetic Biology, pages 59-69. Springer, 2015.

[9] Dawn T Eriksen, Jiazhang Lian, and Huimin Zhao. Protein design for pathway engineering. Journal of Structural Biology, 185(2):234-242, 2014.

[10] Kristala L Jones, Seon-Won Kim, and JD Keasling. Low-copy plasmids can perform as well as or better than high-copy plasmids for metabolic engineering of bacteria. Metabolic Engineering, 2(4):328-338, 2000.

[11] Claes Gustafsson, Sridhar Govindarajan, and Jeremy Minshull. Codon bias and heterologous protein expression. Trends in Biotechnology, 22(7):346-353, 2004. 
[12] Hal Alper, Curt Fischer, Elke Nevoigt, and Gregory Stephanopoulos. Tuning genetic control through promoter engineering. Proceedings of the National Academy of Sciences, 102(36):12678-12683, 2005.

[13] Howard M Salis, Ethan A Mirsky, and Christopher A Voigt. Automated design of synthetic ribosome binding sites to control protein expression. Nature Biotechnology, 27(10):946-950, 2009.

[14] Christian Jäckel, Peter Kast, and Donald Hilvert. Protein design by directed evolution. Annual Reviews Biophysics, 37:153-173, 2008.

[15] Philip A Romero and Frances H Arnold. Exploring protein fitness landscapes by directed evolution. Nature Reviews Molecular Cell Biology, 10(12):866-876, 2009.

[16] Jiri Damborsky and Jan Brezovsky. Computational tools for designing and engineering enzymes. Current Opinion in Chemical Biology, 19:8-16, 2014.

[17] Richard J Fox, S Christopher Davis, Emily C Mundorff, Lisa M Newman, Vesna Gavrilovic, Steven K Ma, Loleta M Chung, Charlene Ching, Sarena Tam, Sheela Muley, John Grate, John Gruber, John Whitman, Roger Sheldon, and Gjalt Huisman. Improving catalytic function by ProSAR-driven enzyme evolution. Nature Biotechnology, 25(3):338-344, 2007.

* One of the premier methods for enhancing protein engineering throughput and effectiveness. 
[18] Kim T Simons, Rich Bonneau, Ingo Ruczinski, and David Baker. Ab initio protein structure prediction of CASP III targets using ROSETTA. Proteins: Structure, Function, and Bioinformatics, 37(S3):171-176, 1999.

**The initial demonstration of ROSETTA, a key de novo protein design tool that has become increasingly effective in designing artificial proteins with predicted activities.

[19] Christopher R Otey, Marco Landwehr, Jeffrey B Endelman, Kaori Hiraga, Jesse D Bloom, and Frances H Arnold. Structure-guided recombination creates an artificial family of cytochromes P450. PLoS Biology, 4(5):e112, 2006.

[20] Effendi Leonard, Parayil Kumaran Ajikumar, Kelly Thayer, Wen-Hai Xiao, Jeffrey D Mo, Bruce Tidor, Gregory Stephanopoulos, and Kristala LJ Prather. Combining metabolic and protein engineering of a terpenoid biosynthetic pathway for overproduction and selectivity control. Proceedings of the National Academy of Sciences, 107(31):13654-13659, 2010.

[21] Sabine Bastian, Xiang Liu, Joseph T Meyerowitz, Christopher D Snow, Mike MY Chen, and Frances H Arnold. Engineered ketol-acid reductoisomerase and alcohol dehydrogenase enable anaerobic 2-methylpropan-1-ol production at theoretical yield in Escherichia coli. Metabolic Engineering, 13(3):345-352, 2011.

[22] Zhi Liu, Kannan Gunasekaran, Wei Wang, Vladimir Razinkov, Laura Sekirov, Esther Leng, Heather Sweet, Ian Foltz, Monique Howard, Anne-Marie Rousseau, Carl Kozlosky, William Fanslow, and Wei Yan. Asymmetrical fc engineering 
greatly enhances antibody-dependent cellular cytotoxicity (adcc) effector function and stability of the modified antibodies. Journal of Biological Chemistry, 289(6):3571-3590, 2014.

[23] Dalong Zhang, Rui Zhang, Jie Zhang, Liying Chen, Chunxia Zhao, Wenyue Dong, Qing Zhao, Qiaqing Wu, and Dunming Zhu. Engineering a hydroxysteroid dehydrogenase to improve its soluble expression for the asymmetric reduction of cortisone to $11 \beta$-hydrocortisone. Applied Microbiology and Biotechnology, 98(21):8879-8886, 2014.

[24] Marnix H Medema, Renske van Raaphorst, Eriko Takano, and Rainer Breitling. Computational tools for the synthetic design of biochemical pathways. Nature Reviews Microbiology, 10(3):191-202, 2012.

[25] Vassily Hatzimanikatis, Chunhui Li, Justin a Ionita, Christopher S Henry, Matthew D Jankowski, and Linda J Broadbelt. Exploring the diversity of complex metabolic networks. Bioinformatics, 21(8):1603-9, April 2005.

[26] Kent McClymont and Orkun S. Soyer. Metabolic tinker: An online tool for guiding the design of synthetic metabolic pathways. Nucleic Acids Research, 41(11), 2013.

[27] Chih Hung Chou, Wen Chi Chang, Chih Min Chiu, Chih Chang Huang, and Hsien Da Huang. FMM: A web server for metabolic pathway reconstruction and comparative analysis. Nucleic Acids Research, 37(SUPPL. 2):129-134, 2009.

[28] Guillermo Rodrigo, Javier Carrera, Kristala Jones Prather, and Alfonso 
Jaramillo. DESHARKY: Automatic design of metabolic pathways for optimal cell growth. Bioinformatics, 24(21):2554-2556, 2008.

[29] James Law, Zsolt Zsoldos, Aniko Simon, Darryl Reid, Yang Liu, Sing Yoong Knew, a. Peter Johnson, Sarah Major, Robert a. Wade, and Howard Y. Ando. Route designer: A retrosynthetic analysis tool utilizing automated retrosynthetic rule generation. Journal of Chemical Information and Modeling, 49(3):593-602, 2009.

[30] Harry Yim, Robert Haselbeck, Wei Niu, Catherine Pujol-Baxley, Anthony Burgard, Jeff Boldt, Julia Khandurina, John D Trawick, Robin E Osterhout, Rosary Stephen, Jazell Estadilla, Sy Teisan, H Brett Schreyer, Stefan Andrae, Tae Hoon Yang, Sang Yup Lee, Mark J Burk, and Stephen Van Dien. Metabolic engineering of Escherichia coli for direct production of 1,4-butanediol. Nature Chemical Biology, 7(7):445-452, 2011.

* The authors developed, implemented, and validated a novel biosynthetic pathway to economically produce 1,4-butanediol, which is not naturally produced by any known organism. Their approach demonstrate the utility of the current generation of pathway design tools.

[31] Ayoun Cho, Hongseok Yun, Jin Hwan Park, Sang Yup Lee, and Sunwon Park. Prediction of novel synthetic pathways for the production of desired chemicals. BMC Systems Biology, 2010.

[32] Harris H Wang, Farren J Isaacs, Peter A Carr, Zachary Z Sun, George Xu, 
Craig R Forest, and George M Church. Programming cells by multiplex genome engineering and accelerated evolution. Nature, 460(7257):894-898, 2009.

[33] Ramsey I Zeitoun, Andrew D Garst, George D Degen, Gur Pines, Thomas J Mansell, Tirzah Y Glebes, Nanette R Boyle, and Ryan T Gill. Multiplexed tracking of combinatorial genomic mutations in engineered cell populations. Nature Biotechnology, 2015.

[34] Jina Yang, Sang Woo Seo, Sungho Jang, So-I Shin, Chae Hyun Lim, Tae-Young Roh, and Gyoo Yeol Jung. Synthetic RNA devices to expedite the evolution of metabolite-producing microbes. Nature Communications, 4:1413, 2013.

*The authors develop a selection for amino acid production by coupling a riboswitch with a positive and negative selectable marker, allowing dramatic increases in productivity without relying on rational approaches.

[35] William R Farmer and James C Liao. Improving lycopene production in escherichia coli by engineering metabolic control. Nature Biotechnology, 18(5):533$537,2000$.

[36] Fuzhong Zhang, James M Carothers, and Jay D Keasling. Design of a dynamic sensor-regulator system for production of chemicals and fuels derived from fatty acids. Nature Biotechnology, 30(4):354-359, 2012.

[37] Parayil Kumaran Ajikumar, Wen-Hai Xiao, Keith EJ Tyo, Yong Wang, Fritz Simeon, Effendi Leonard, Oliver Mucha, Too Heng Phon, Blaine Pfeifer, and 
Gregory Stephanopoulos. Isoprenoid pathway optimization for Taxol precursor overproduction in Escherichia coli. Science, 330(6000):70-74, 2010.

[38] Igor Ulitsky and Ron Shamir. Identifying functional modules using expression profiles and confidence-scored protein interactions. Bioinformatics, 25(9):11581164, 2009.

[39] Nicolas Alcaraz, Hande Kücük, Jochen Weile, Anil Wipat, and Jan Baumbach. KeyPathwayMiner: detecting case-specific biological pathways using expression data. Internet Mathematics, 7(4):299-313, 2011.

[40] Yanpeng Hou, Doug R Braun, Cole R Michel, Jonathan L Klassen, Navid Adnani, Thomas P Wyche, and Tim S Bugni. Microbial strain prioritization using metabolomics tools for the discovery of natural products. Analytical Chemistry, 84(10):4277-4283, 2012.

[41] Keesha E Erickson, Ryan T Gill, and Anushree Chatterjee. CONSTRICTOR: Constraint Modification Provides Insight into Design of Biochemical Networks. PloS ONE, 9(11):e113820, 2014.

[42] Laurence Yang, William R Cluett, and Radhakrishnan Mahadevan. EMILiO: a fast algorithm for genome-scale strain design. Metabolic Engineering, 13(3):272$281,2011$.

[43] Anupam Chowdhury, Ali R Zomorrodi, and Costas D Maranas. k-OptForce: integrating kinetics with flux balance analysis for strain design. PLoS Computational Biology, 10(2):e1003487, 2014. 
[44] Brooks B Bond-Watts, Robert J Bellerose, and Michelle CY Chang. Enzyme mechanism as a kinetic control element for designing synthetic biofuel pathways. Nature Chemical Biology, 7(4):222-227, 2011.

[45] Amarjeet Singh, Michael D Lynch, and Ryan T Gill. Genes restoring redox balance in fermentation-deficient e. coli nzn111. Metabolic Engineering, 11(6):347$354,2009$.

[46] Yun Chen, Laurent Daviet, Michel Schalk, Verena Siewers, and Jens Nielsen. Establishing a platform cell factory through engineering of yeast acetyl-coa metabolism. Metabolic Engineering, 15:48-54, 2013.

[47] Mary J Dunlop, Zain Y Dossani, Heather L Szmidt, Hou Cheng Chu, Taek Soon Lee, Jay D Keasling, Masood Z Hadi, and Aindrila Mukhopadhyay. Engineering microbial biofuel tolerance and export using efflux pumps. Molecular Systems Biology, 7(1), 2011.

[48] James D Winkler, Carlos Garcia, Michelle Olson, Emily Callaway, and Katy C Kao. Evolved Osmotolerant Escherichia coli Mutants Frequently Exhibit Defective N-Acetylglucosamine Catabolism and Point Mutations in Cell Shape-Regulating Protein MreB. Applied and Environmental Microbiology, 80(12):3729-3740, 2014.

[49] Michael D Lynch, Tanya Warnecke, and Ryan T Gill. SCALEs: multiscale analysis of library enrichment. Nature Methods, 4(1):87-93, 2007.

[50] Leqian Liu, Anny Pan, Caitlin Spofford, Nijia Zhou, and Hal S Alper. An evo- 
lutionary metabolic engineering approach for enhancing lipogenesis in Yarrowia lipolytica. Metabolic Engineering, 29:36-45, 2015.

[51] Florence Mingardon, Camille Clement, Kathleen Hirano, Melissa Nhan, Eric G Luning, Angelique Chanal, and Aindrila Mukhopadhyay. Improving olefin tolerance and production in E. coli using native and evolved AcrB. Biotechnology and Bioengineering, 2015.

[52] Jessica S Dymond, Sarah M Richardson, Candice E Coombes, Timothy Babatz, Héloïse Muller, Narayana Annaluru, William J Blake, Joy W Schwerzmann, Junbiao Dai, Derek L Lindstrom, Annabel C Boeke, Gottschling Daniel E, Srinivasan Chandrasegaran, Joel S. Bader, and Jef D Boeke. Synthetic chromosome arms function in yeast and generate phenotypic diversity by design. Nature, 477(7365):471-476, 2011.

[53] Jonathan R Karr, Jayodita C Sanghvi, Derek N Macklin, Miriam V Gutschow, Jared M Jacobs, Benjamin Bolival, Nacyra Assad-Garcia, John I Glass, and Markus W Covert. A whole-cell computational model predicts phenotype from genotype. Cell, 150(2):389-401, 2012.

One of the most complete cellular models currently available, the Mycobacterium cell model developed in this study was able to predict novel biological behaviors arising from subsystem interconnectivity in vivo.

[54] M Tomita, K Hashimoto, K Takahashi, T S Shimizu, Y Matsuzaki, F Miyoshi, 
K Saito, S Tanida, K Yugi, J C Venter, and C A Hutchison. E-CELL: software environment for whole-cell simulation. Bioinformatics, 15(1):72-84, 1999.

[55] Jeffrey A Dietrich, Adrienne E McKee, and Jay D Keasling. High-throughput metabolic engineering: advances in small-molecule screening and selection. Annual Review of Biochemistry, 79:563-590, 2010.

[56] Horst Zuse. Software complexity. NY, USA: Walter de Cruyter, 1991.

[57] Thomas J McCabe. A complexity measure. IEEE Transactions on Software Engineering, (4):308-320, 1976.

[58] Maurice H Halstead. Toward a theoretical basis for estimating programming effort. In Proceedings of the 1975 annual conference, pages 222-224. ACM, 1975 .

[59] Warren Harrison, Kenneth Magel, Raymond Kluczny, and Arlan DeKock. Applying software complexity metrics to program maintenance. Computer, 15(9), 1982.

[60] Don Coleman, Dan Ash, Bruce Lowther, and Paul Oman. Using metrics to evaluate software system maintainability. Computer, 27(8):44-49, 1994.

[61] Bill Curtis, Sylvia B Sheppard, and Phil Milliman. Third time charm: Stronger prediction of programmer performance by software complexity metrics. In Proceedings of the 4th international conference on Software engineering, pages 356360. IEEE Press, 1979. 
[62] Taghi M. Khoshgoftaar and John C. Munson. Predicting software development errors using software complexity metrics. IEEE Journal on Selected Areas in Communications, 8(2):253-261, 1990.

[63] Yonghee Shin, Andrew Meneely, Laurie Williams, and Jason A Osborne. Evaluating complexity, code churn, and developer activity metrics as indicators of software vulnerabilities. IEEE Transactions on Software Engineering, 37(6):772787, 2011.

[64] James D Winkler, Andrea L Halweg-Edwards, and Ryan T Gill. The LASER database: Formalizing design rules for metabolic engineering. Metabolic Engineering Communications, 2:30-38, 2015. 


\section{Figures}

Figure 1: Distribution of methods used to engineer existing metabolically engineered strains [64]. Legend: HUM, human inference using knowledge or past experience, such as manually examining the metabolic network and selecting mutational targets; LIB, library methods; FBA, flux balance analysis; EVO, adaptive evolution for production or tolerance; RAND, random protein or genome mutagenesis; PREN: targeted protein engineering, TRANS, transcriptomics; LL, liquid-liquid (twophase) product extraction; COMP, other computational methods; OTH, other approaches.

Figure 2: How protein engineering methods compare in terms of algorithmic complexity and library coverage. Random methods require little (if any) knowledge of the protein of interest but require much larger library sizes to progress through the protein fitness landscape; computational tools are able to reduce the required library size for screening but require substantially more sophisticated algorithms and training datasets to function properly.

Figure 3: Progression in complexity for pathway-level design in metabolic engineering. Initial screens for potential enzymes using high-throughput techniques (metabolomics) that furnish many potentially useful enzymatic reactions; these enzymes can be cloned and expressed using metagenomic libraries. As models become more sophisticated, this random approach can be superseded by metabolic network modeling and database searches of known or predicted pathways. 
Figure 4: Complexity trends for genome-level design methods. Random methods, such as mutagenesis and adaptive evolution, are frequently employed in the absence of genotype-phenotype connections. Rational design can be applied if sufficient knowledge and metabolic models are available for the organism in question. Future developments in whole genome refactoring and complete synthesis require significant improvements in our understanding of genome architecture but promise to enable unique engineering approaches. 


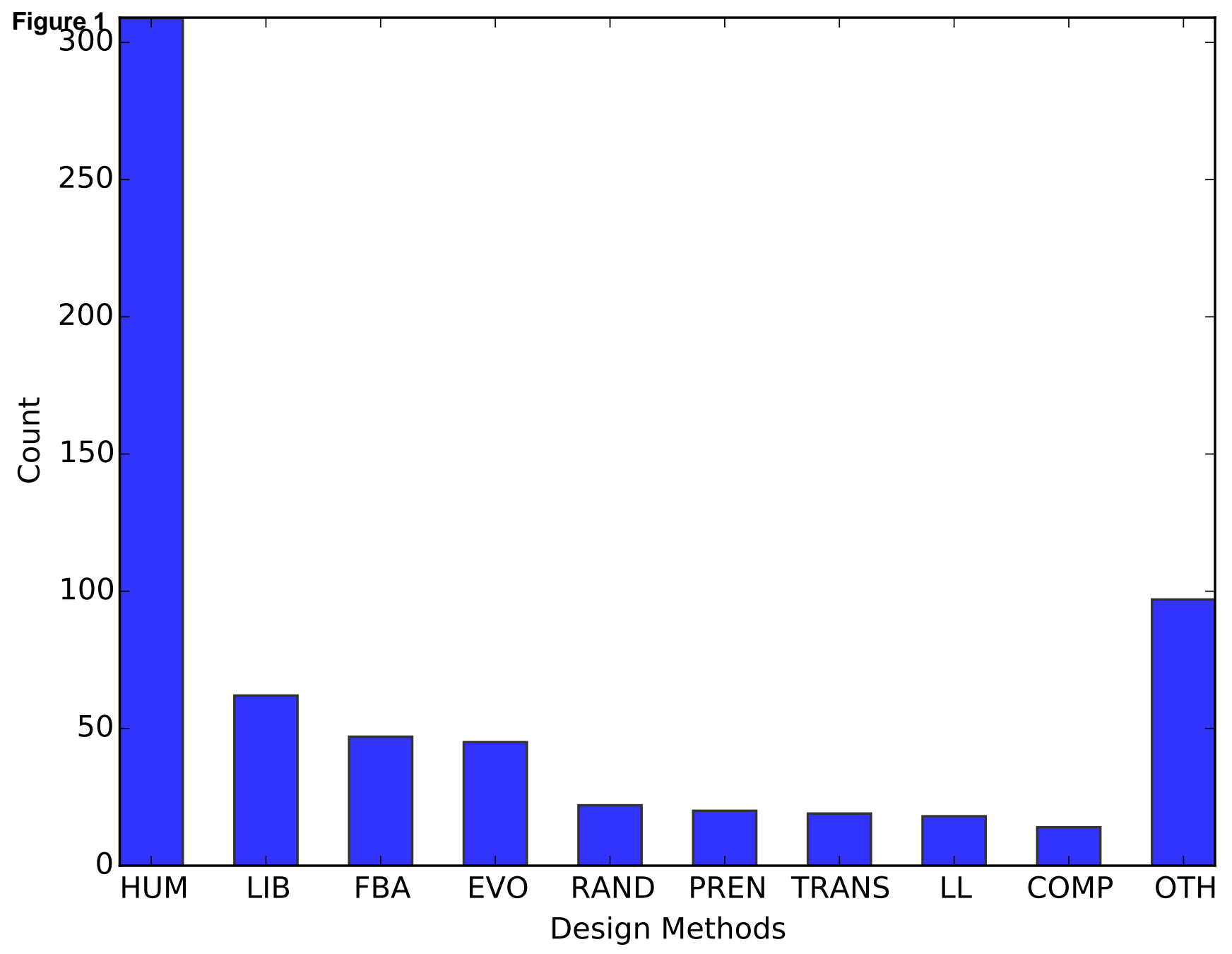


Random Mutagenesis

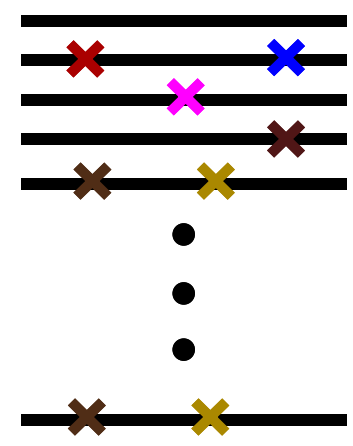

Statistical Modeling

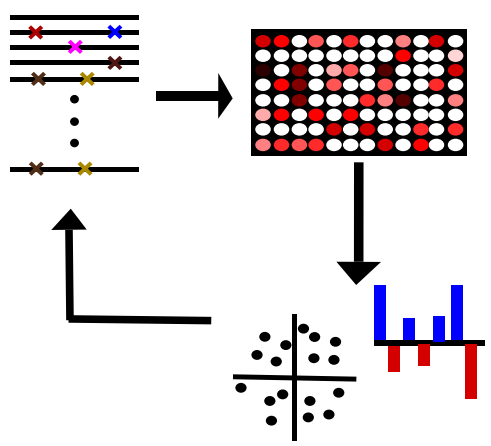

Sequence homology based approaches

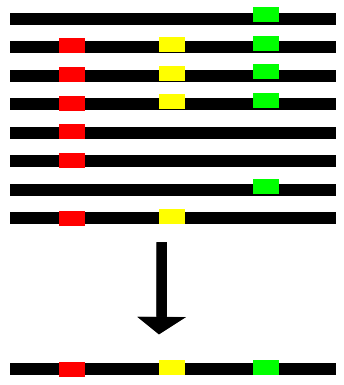

Rational modular design

Algorithmic Complexity and Computation Time 
Meta-Metabolomics

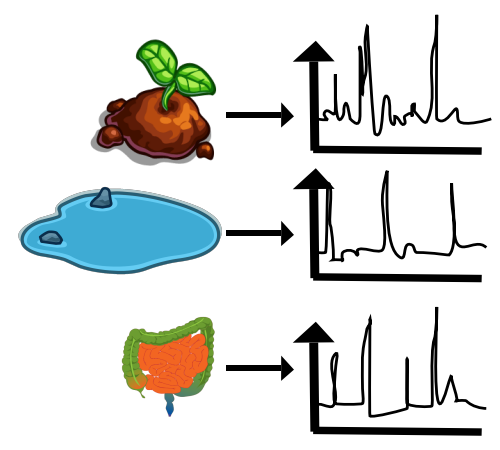

Network Modeling

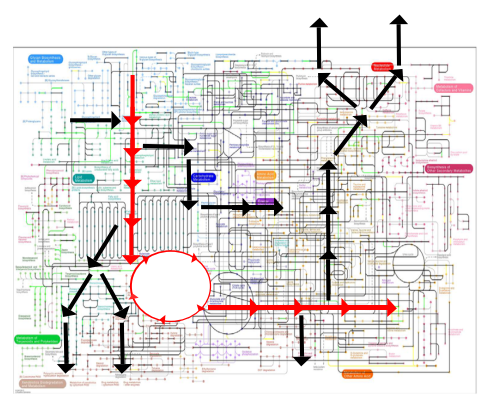

Pathway Search Algorithms

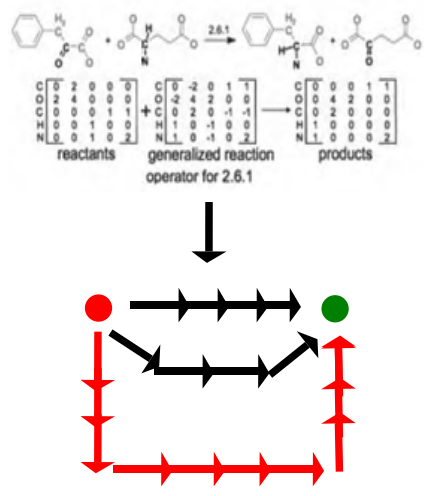

Algorithmic Complexity and Computation Time 
Adaptive Evolution

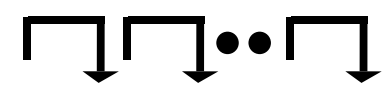

bation

000
Rational Design

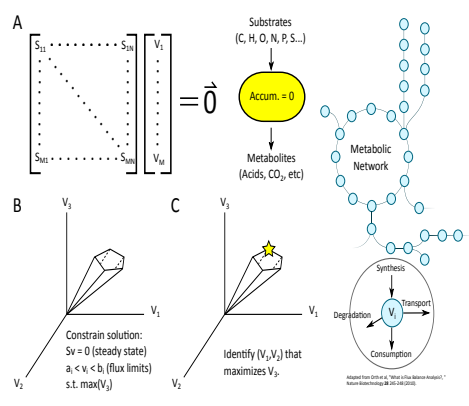

Genome Refactoring
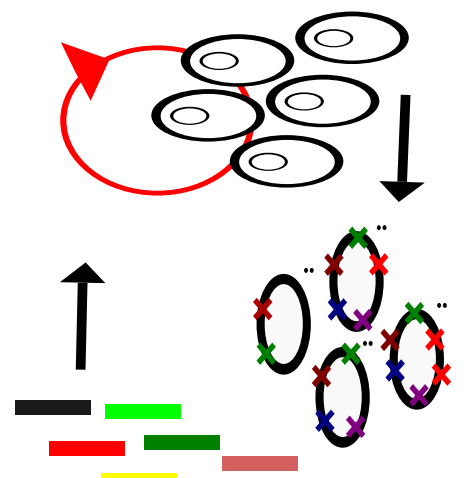

Whole Genome Redesign/ Genome Synthesis

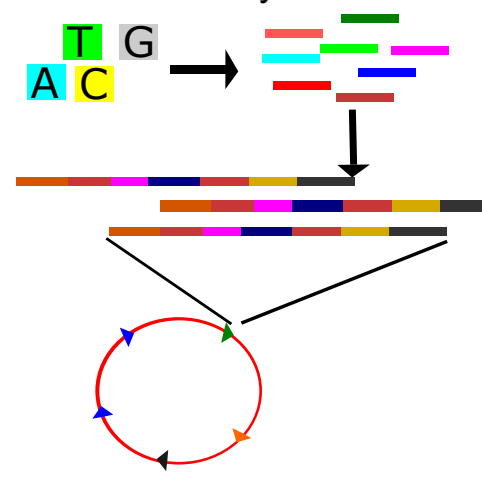

Algorithmic Complexity and Computation Time 